\title{
Pulmonary Gas Exchange During Hemodialysis: A Comparison of Subjects with and without COPD on Bicarbonate Hemodialysis
}

\author{
Maria Alfakir ${ }^{1,2,4}$, Mahmoud Q. Moammar 1,2,4, Muhammad I. Ali ${ }^{1,2,4}$, Eid Alhatem ${ }^{1}$, Ryan D. Curran ${ }^{5}$, \\ Rani M. Saoud ${ }^{5}$, Chandra Chandran ${ }^{1,3}$, M Anees Khan ${ }^{1,2,4}$ and Vincent A. DeBari ${ }^{1,4}$ \\ Departments of ${ }^{1}$ Medicine and Divisions of ${ }^{2}$ Pulmonary Diseases and ${ }^{3}$ Nephrology, St. Joseph's Regional Medical Cen- \\ ter, Paterson, NJ, USA; ${ }^{4}$ School of Health and Medical Sciences, Seton Hall University, South Orange, NJ, USA; \\ ${ }^{5}$ St.George's University School of Medicine, Grenada, W.I.
}

\begin{abstract}
Little is known about the effect of hemodialysis (HD) on gas exchange in subjects with chronic obstructive pulmonary disease (COPD). The purpose of this study was to examine blood gases and $\mathrm{pH}$ in COPD patients undergoing HD with bicarbonate dialysate. We studied thirteen subjects with COPD and thirteen controls $(\mathrm{CON})$. All were dialyzed for $4 \mathrm{hrs}$ against a bicarbonate $\mathrm{HD}$ solution. Blood gases, $\mathrm{pH}$ and $\mathrm{HCO}_{3}{ }^{-}$were initially analyzed $\left(\mathrm{t}_{0}\right)$ and, during $\mathrm{HD}$, at $30 \mathrm{~min}\left(\mathrm{t}_{0.5}\right), 1 \mathrm{hr}\left(\mathrm{t}_{1}\right)$ and $4 \mathrm{hrs}\left(\mathrm{t}_{4}\right)$. At $\mathrm{t}_{0}$, a statistically significant difference was observed for $\mathrm{PO}_{2}(\mathrm{CON}: 84.7 \pm 3.60$, COPD: $72.19 \pm 4.92 ; \mathrm{p}<0.001)$. For the first $\mathrm{hr}, \mathrm{PO}_{2}$ decreased, and at $\mathrm{t}_{1}$, oxygen was required for $6 \mathrm{COPD}$ subjects. $\mathrm{By}_{4}$, there was no significant difference in $\mathrm{PO}_{2}$ between groups. The alveolar-arterial gradient $(\triangle \mathrm{A}-\mathrm{a})$ remained different between groups ( $\mathrm{P}<0.001$, all times), with increasing $\triangle \mathrm{A}$-a for both groups up to $\mathrm{t}_{1}$ and decreasing over the remaining $3 \mathrm{hr}$. For both groups, at $\mathrm{t}_{4}, \Delta \mathrm{A}$-a was higher than at $\mathrm{t}_{0}(\mathrm{p}<0.001)$. For $\mathrm{PCO}_{2}$, both groups demonstrated increases from $\mathrm{t}_{0}$ to $\mathrm{t}_{1}(\mathrm{p}=0.0004)$, with COPD having $\mathrm{PCO}_{2}$ higher than $\mathrm{CON}$ at $\mathrm{t}_{0.5}$ and $\mathrm{t}_{1}\left(\mathrm{p}<0.05\right.$ for both); by $\mathrm{t}_{4}, \mathrm{PCO}_{2}$ levels decreased to nearly the same as at $\mathrm{t}_{0}$. Over the $4 \mathrm{hr}$ treatment, $\mathrm{HCO}_{3}{ }^{-}$and $\mathrm{pH}$ increased significantly for both groups; however no significant difference was observed between COPD and CON. Markedly increased $\triangle \mathrm{A}-\mathrm{a}$ is observed during HD in some COPD patients. COPD patients retain more $\mathrm{CO}_{2}$. However, the effect of $\mathrm{HCO}_{3}{ }^{-}$leads to mild metabolic alkalosis at $\mathrm{t}_{4}$.
\end{abstract}

Key words: Bicarbonate hemodialysis; acid-base equilibrium; chronic obstructive pulmonary disease; pulmonary gas exchange; blood gases.

Introduction

Hypoxemia during hemodialysis (HD) is a welldescribed phenomenon [1-5]. Although several mechanisms have been proposed [6], the cause remains unclear. Some studies have attributed hypoxemia during HD to the type of dialysate [7], to the type of membrane used [8], or to both [9-11]. Acetate dialysate, for example, has been found to trigger hypoxemia by the loss of carbon dioxide $\left(\mathrm{CO}_{2}\right)$ across the dialyzer $[12,13]$. This loss in $\mathrm{CO}_{2}$ results in alveolar hypoventilation. Ventilation/ perfusion (V/Q) mismatch has been proposed as the cause of hypoxemia by Craddok et al. [14] and

Address correspondence to Vincent A. DeBari, PhD, Department of Medicine, School of Health and Medical Sciences, Seton Hall University, South Orange, NJ, 07079 USA
Bischel et al. [15]. It has also been suggested that the activation of the complement system by the non-biocompatible dialysis membrane triggers pulmonary leukostasis [14].

As the cause of hypoxemia during HD remains under debate, the effects of this repetitive transient hypoxemia on chronic obstructive pulmonary disease (COPD) patients should also be considered. As this minor degree of hypoxemia may remain tolerable in normal subjects, patients with pulmonary compromise may not be able to tolerate it over an extended period of treatment. In fact, this hypoxemia may well contribute to these patients' morbidity and even mortality. 
Therefore, we have elected to study the effects of bicarbonate-based HD on gas exchange in patients with COPD and to compare them to the effects on subjects without COPD. The purpose of our study is to examine the abnormalities of gas exchange that take place in COPD patients.

\section{Materials and Methods}

Patients and Protocol. A cohort consisting of twentysix HD patients was divided into two groups: those with a diagnosis of COPD, based on the criteria of the Global Initiative for Chronic Obstructive Lung Disease for all classes of COPD, i.e., $\mathrm{FEV}_{1} / \mathrm{FVC}<70$ [16], and a control group that had no clinical evidence of COPD. Of 265 subjects screened for inclusion, 13 were determined to have COPD; the age and sex-matched controls were sequentially chosen from the remaining subjects without COPD. In each group, there were 8 males and 5 females. All of the 13 patients with COPD were smokers while 4 of the 13 controls were non-smokers. The patients were included in the study if they were over 21 years of age, underwent dialysis via established arteriovenous $(\mathrm{A}-\mathrm{V})$ fistulae, and were on dialysis for more than 6 months. Patients were excluded if they did not meet all the inclusion criteria, or if they had chronic respiratory failure requiring the use of continuous or intermittent oxygen therapy, or had a baseline resting $\mathrm{PO}_{2}$ of less than $60 \mathrm{mmHg}$ on room air. Because patients were required to have been on maintenance dialysis for a minimum of six months, fistulae were considered to be fully arterialized.

This observational study examined changes in $\mathrm{PO}_{2}$, $\mathrm{PCO}_{2}, \mathrm{pH}$, and $\mathrm{HCO}_{3}{ }^{-}$and the alveolar-arterial oxygen gradient, $\triangle \mathrm{A}$-a. Arterial blood was drawn from the A-V fistula for the first specimen immediately before the initiation of HD. The second and third specimens were drawn from the A-V fistula at 30 and $60 \mathrm{~min}$, respectively, after the initiation of HD. The fourth specimen was drawn at $240 \mathrm{~min}$, immediately before the termination of HD.

The study was approved by Institutional Review Board at St. Joseph's Regional Medical Center.

O2 Supplementation. In cases where blood $\mathrm{PO}_{2}$ was found to drop significantly during or after $\mathrm{HD}$, the patients were provided with supplemental $\mathrm{O}_{2}$ according to the following algorithm: For COPD patients: the supplementation threshold was a $\mathrm{PO}_{2} \leq 60 \mathrm{mmHg}$; for an individual without known underlying lung disease, a
$\mathrm{PO}_{2}<70 \mathrm{mmHg}$ was considered the threshold for $\mathrm{O}_{2}$ supplementation. Supplementation with $\mathrm{O}_{2}$ was done via nasal cannula.

Dialysis Procedure. Therapy sessions with HD were performed using Fresenius $\mathrm{K}$ machines. All the subjects were dialyzed with a bicarbonate dialysate bath $\left(\mathrm{HCO}_{3}{ }^{-}\right.$concentration at our standard protocol of 37 mEq per liter) in which the acetate concentration was 4 $\mathrm{mEq} / \mathrm{l}$. The dialyzers were Fresenius Optiflux using new polysulfone membranes. AV fistulas were used for cannulation with either 14 or 15 gauge needles. Blood flow was maintained at $300-400 \mathrm{ml} / \mathrm{min}$.

Patients were studied for two dialysis sessions; the mean of the two values for each patient were used. The session to session values never varied by more than ten percent.

Blood Gas Analysis. Blood samples, taken from the A-V fistula within $6 \mathrm{~cm}$ of the anastomotic site to minimize venous mixing, were drawn into syringes that were immediately capped and placed on an ice bath. The $\mathrm{PO}_{2}, \mathrm{PCO}_{2}, \mathrm{pH}$, and $\mathrm{HCO}_{3}{ }^{-}$were analyzed on a $\mathrm{Gem}^{\circ}$ Premier 2000 Blood Gas Analyzer (Instrumentation Laboratory (Lexington MA, USA) 5-10 minutes after being drawn. All analyses were within acceptable analytical standards for the instrument controls. The $\Delta \mathrm{A}-\mathrm{a}$ was calculated as the difference between the alveolar $\mathrm{PO}_{2}$ and arterial $\mathrm{PO}_{2}$, with alveolar $\mathrm{O}_{2}$ concentration calculated from the formula: $\mathrm{P}_{\mathrm{AO} 2}=[$ Fractional concentration of inhaled oxygen $\mathrm{x}$ (barometric pressure partial pressure of water vapor in air)] $-\mathrm{Pa}_{\mathrm{CO}} / 0.8$.

Statistical Analysis. For this study, $\alpha$ was set at 0.05 ; comparisons for which $\mathrm{p}<0.05$ (two-sided) were considered to demonstrate significant differences between groups. Categorical variables were compared using cross-tabulations and analyzed for statistical significance by Fisher's exact test. Continuous variables were tested for fit-to-normality with the D'Agostino-Pearson omnibus normality test. Because all but three groups $\left(\mathrm{PO}_{2}, 30\right.$ min. COPD group, $\mathrm{PO}_{2}, 240 \mathrm{~min}$. Control group, and $\triangle \mathrm{A}-\mathrm{a}$, initial, COPD group) were normally distributed, parametric methods were used, with data expressed throughout as means \pm 1 standard deviation (SD). A two-way ANOVA model for each dependent variable $\left(\mathrm{PO}_{2}, \triangle \mathrm{A}-\mathrm{a}, \mathrm{PCO}_{2}, \mathrm{pH}\right.$, and $\left.\mathrm{HCO}_{3}^{-}\right)$considered two independent variables: (1) group as an independently assorted measure and (2) time, as a repeated measure.

Data were analyzed using Prism ${ }^{\circ}$ software (GraphPad Corp., San Diego CA, USA) on a personal computerWindows ${ }^{\oplus}$ Vista $^{\oplus}$ platform. 
Table 1. Baseline characteristics of the study groups. Categorical variables other than gender are presented as those with/ those without the indicated condition.

\begin{tabular}{llll}
\hline Characteristic & COPD & No COPD & p-value \\
\hline Age, yrs (mean \pm SD) & $63.1 \pm 10.4$ & $61.2 \pm 15.4$ & 0.724 \\
Gender (M/F) & $8 / 5$ & $8 / 5$ & 1.00 \\
BMI (mean \pm SD) & $24.7 \pm 3.78$ & $22.3 \pm 3.46$ & 0.151 \\
Hypertension & $10 / 3$ & $8 / 5$ & 0.673 \\
Diabetes & $5 / 8$ & $7 / 6$ & 0.695 \\
Dyslipidemia & $4 / 9$ & $6 / 7$ & 0.688 \\
Coronary artery disease & $2 / 11$ & $4 / 9$ & 0.645 \\
Cardiomyopathy & $3 / 10$ & $1 / 12$ & 0.593 \\
Peripheral vascular disease & $1 / 12$ & $3 / 10$ & 0.593 \\
Systemic lupus erythematosus & $0 / 13$ & $1 / 12$ & 1.00 \\
Rheumatoid arthritis & $0 / 13$ & $1 / 12$ & 1.00 \\
Hepatitis B & $1 / 12$ & $0 / 13$ & 1.00 \\
Hepatitis C & $1 / 12$ & $1 / 12$ & 1.00 \\
HIV disease & $0 / 13$ & $1 / 12$ & 1.00 \\
\hline
\end{tabular}

\section{Results}

Baseline demographics for the two groups are provided in Table 1. There were no significant differences with regard to either basic demographics or co-morbidities.

Parameters relating to oxygenation are shown in Figure 1. The COPD group demonstrated significantly $(\mathrm{p}<0.0001)$ lower initial $\mathrm{PO}_{2}$ and this trend continued over the course of the first hour of dialysis (change over time also statistically significant at $\mathrm{p}<0.0001)$. The apparent increase in $\mathrm{PO}_{2}$ for the COPD subjects is due to their having received $\mathrm{O}_{2}$ supplementation, as described in METHODS. In fact, as can be seen in the plot of $\triangle \mathrm{A}$-a, the differences in that parameter trended $(\mathrm{p}<0.0001)$ in the same direction with a comparably significant difference $(\mathrm{p}<0.0001)$ between the groups.

Figure 2 provides data on $\mathrm{PCO}_{2}, \mathrm{HCO}_{3}{ }^{-}$and $\mathrm{pH}$. Not unexpectedly, initial $\mathrm{PCO}_{2}$ in the COPD group is elevated and remains significantly so $(\mathrm{p}<0.0001)$ over the first hour of HD. By $4 \mathrm{hr}$, the PCO2 diminishes to a value close to the control group; in fact, there was no significant difference observed between the two groups by that time.

A slightly significant difference in $\mathrm{HCO}_{3}{ }^{-}$is seen during the course of the HD treatment overall $(\mathrm{p}=$ 0.026), although post-tests failed to detect a difference at any single time period. The time factor was highly significant $(\mathrm{p}<0.0001)$ as plasma $\mathrm{HCO}_{3}{ }^{-}$reached equilibrium with the $\mathrm{HCO}_{3}{ }^{-}$concentration of the bath.

For $\mathrm{pH}$, although a significant difference $(\mathrm{p}=$ $0.004)$ is observed between the groups, only the difference at $1 \mathrm{hr}$ was detected as significant by post-test. Initially the COPD and control $\mathrm{pH}$ values are quite comparable; however, over the course of the HD treatment both groups trend toward a metabolic alkalosis. This is alleviated, to a degree, in the COPD group, whose PCO2 values increase to demonstrate partial respiratory compensation.

\section{Discussion}

This study demonstrates that hypoxemia during HD occurs in both patients with and without COPD to nearly the same degree. However, due to the fact that patients with COPD have a lower baseline $\mathrm{PO}_{2}$, the nadir $\mathrm{PO}_{2}$ in this group was lower. The comparable values of $\mathrm{PO}_{2}$ that were seen at the end of $\mathrm{HD}$ were due to $\mathrm{O}_{2}$ supplementation to patients with COPD whose $\mathrm{PO}_{2}$ fell below $60 \mathrm{mmHg}$ during the first hour of the HD session. While Serrano et al. showed that the occurrence of hypoxemia during the first hour of HD only in subjects with chronic pulmonary disease 

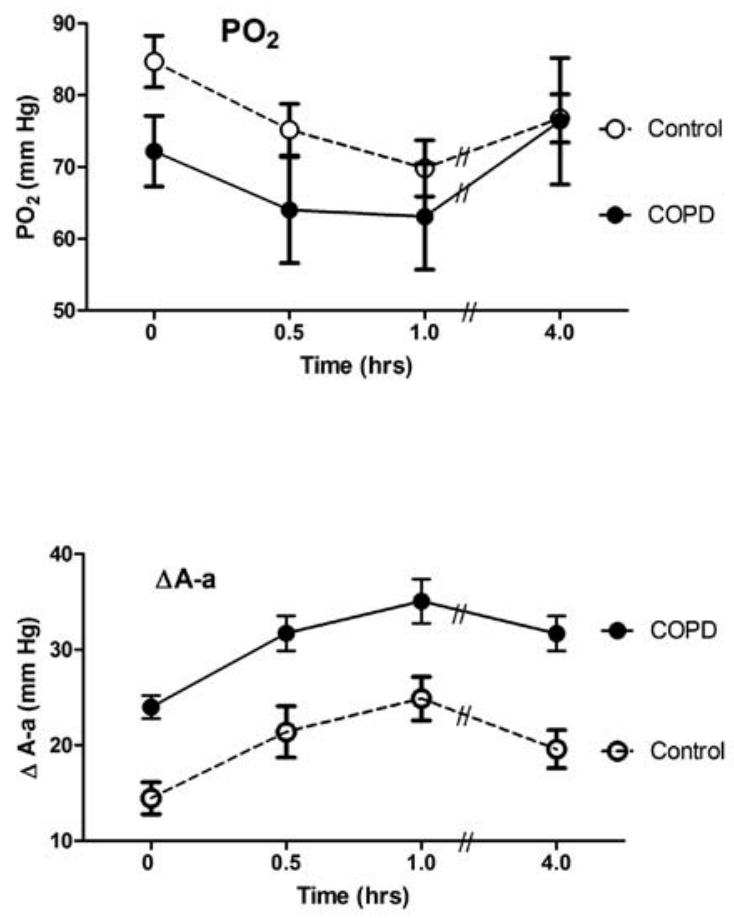

Figure 1. Parameters of blood oxygenation over the course of a four-hour $H D$ treatment. Data are means $\pm 1 S D$

undergoing bicarbonate HD [17], Pitcher et al. demonstrated the occurrence of hypoxemia only at the end of the HD session in subjects with and without COPD [18]. In contrast, our study showed that hypoxemia during bicarbonate HD occurred at 30 minutes, peaked at 60 minutes and continued until the end of the HD session. The cause of this hypoxemia during the first hour of HD is unclear. The minute ventilation during bicarbonate HD was found to be decreased in the control group during the first hour of HD but not to a point reaching statistical significance [18]. Therefore, bicarbonate-induced hypoventilation may be one mechanism that could explain hypoxemia within the first hour of HD. Other proposed theories are V/Q mismatch, activation of complement, and microembolization to the lungs [6]. However, these mechanisms do not explain the persistence of hypoxemia throughout the HD session. Moreover, the use of a polysulfone membrane minimizes the activation of complement and pulmonary leukostasis, thereby minimizing their contribution to the observed degree of hypoxemia [8].

Our data also demonstrate a comparable rise in $\mathrm{PCO}_{2}$ in both COPD and non-COPD subjects. However, because the baseline $\mathrm{PCO}_{2}$ in subjects with COPD is higher than in the control subjects, the nadir $\mathrm{PCO}_{2}$ in subjects with COPD remains higher. These findings are consistent with a previously observed rise in $\mathrm{PCO}_{2}$ during $\mathrm{HD}$ [18]. The rise in $\mathrm{PCO}_{2}$ could be explained theoretically by both hypoventilation and V/Q mismatch. The most likely cause of the decline in $\mathrm{PCO}_{2}$ towards the end of dialysis is the resolution of hypoventilation induced by hypoxemia.

We observed comparable changes in $\mathrm{HCO}_{3}{ }^{-}$in both COPD and non-COPD. Ultimately both groups' plasma $\mathrm{HCO}_{3}{ }^{-}$levels equilibrate to the concentration in the dialysate. A continuous rise in $\mathrm{pH}$ was observed in both the COPD and the control groups. This rise in $\mathrm{pH}$ mirrors the rise in serum bicarbonate in both groups.

In summary this study is the first prospective study done on COPD patients undergoing bicarbonate $\mathrm{HD}$ to observe the effect of $\mathrm{HD}$ on all arterial blood gas components including: $\mathrm{PO}_{2}, \triangle \mathrm{A}$-a, $\mathrm{PCO}_{2}, \mathrm{HCO}_{3}^{-}$and $\mathrm{pH}$. Our data clearly show that hypoxemia during bicarbonate $\mathrm{HD}$ takes place within 30 minutes of initiation of HD and peaks at 60 minutes in both COPD and non-COPD subjects. To our knowledge this has not been previously demonstrated. Serrano et al. demonstrated hypoxemia at 30 and 60 minutes in the chronic lung disease group only and failed to detect any hypoxemia in the control group at the aforementioned intervals [17]. Pitcher et al. did not demonstrate any hypoxemia in either the control or the COPD groups at one-hour post initiation of HD [18]. In contrast, our study showed that hypoxemia occurs within the first hour and continues throughout the HD session.

The fact that hypoxemia continues throughout the HD session leaving the COPD subjects with a higher $\Delta \mathrm{A}-\mathrm{a}$ at the end of the session than at the initiation of the HD session shows the necessity of supplementing these patients with oxygen during and after HD. In fact, oxygen supplementation in these patients may eliminate the consequences of repetitive hypoxemia such as arrhythmias and acceleration of the development of pulmonary hypertension. Moreover, our data, when examined in light of data on other membrane and buffer systems, suggest that there may be cause to examine HD patients more routinely for V/Q mismatch as evidenced by increased A-a gradients. 

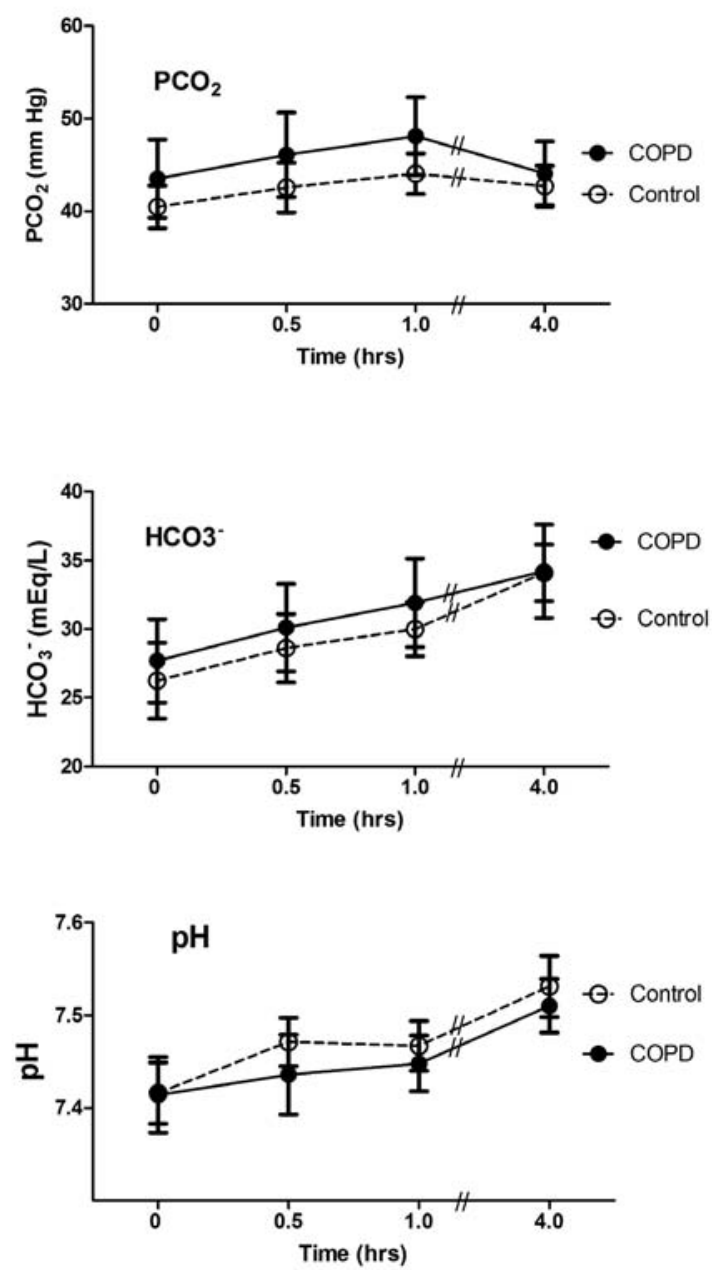

Figure 2. Acid-base parameters $\left(\mathrm{PCO}_{2}, \mathrm{HCO}_{3}\right.$, and $\left.\mathrm{PH}\right)$ during $\mathrm{HD}$. Data expressed as in the previous figure.

Alkalosis is another factor that may contribute to morbidity and mortality in patients on HD. In our study a significant degree of alkalosis was observed among patients, especially at the end of HD session, as a considerable number of subjects had a $\mathrm{pH}$ above 7.5 , the result of using the bicarbonate dialysate. The effects of this alkalosis on these patients and methods of eliminating it certainly bear further investigation. Recent data suggest that there may be higher incidence of deaths on Mondays and Tuesdays after the two-day gap in HD treatments. Although it is purely hypothetical, it is tempting to speculate that this increased mortality may be related to the initial acidosis and rapid correction of $\mathrm{pH}$ to the alkalotic side with concomitant electrolyte shift and arrhythmias [191]. This also needs to be further explored, especially in that arterial blood is easily accessed in this group of subjects obviating the need for direct arterial puncture.

We recognize that our study has some limitations. We studied only thirteen subjects in each group. This is unlikely to have been problematic in terms of the primary dependent variables, as the withingroup dispersion for each variable (Figures 1 and 2) can be observed to be relatively small. However the baseline characteristics (Table 1) include several comparisons for which statistical analysis suggests that there is no difference between groups when, in fact, this may represent a Type 2 testing error, i.e., we may not have had adequate power to detect a difference.

\section{Acknowledgement}

We would like to thank the nursing staff at the hemodialysis center at St. Joseph Regional Medical Center and Jeffery Zdanewicz, Rachel Ruf and Juan Rivera for all their help and cooperation.

\section{References}

1. Habte B, Carter R, Shamebo M, Veicht J, Boulton Jones JM. Dialysis Induced Hypoxemia. Clinical Nephrology. 1982 Sep; 18(3): 120-125.

2. Kishimoto T, Tanaka H, Maekawa M, Ivanovich P, Levin N, Bergstrom J, Klinkmann H. Dialysis-Induced Hypoxemia. Nephrol Dial Transplant. 1993; 8 [Supp.2]: 25-29.

3. De Broe ME. Hemodialysis-Induced Hypoxemia. Nephrol Dial Transplant. 1994; 9 [Supp. 2] : 173-175.

4. Fawcett S, Hoenich NA, Laker MF, Schorr Jr W, Ward, MK, Kerr DNS. Hemodialysis-Induced Respiratory Changes. Nephrol Dial Transplant 1987; 2: 161-168.

5. Garella S, Chang BS. Hemodialysis-Associated Hypoxemia. Am. J. Nephrol 1984; 4: 273-279.

6. De Broe ME, Heyrman, RM, De Backer WA, Verpooten GA, Vermeire PA. Pathogenesis of Dialysis-Induced Hypoxemia: A Short Overview. Kidney International, 1988: 33(24): S57-S61.

7. Abu-Hamdan DK, Desai SG, Mahajan SK, Muller BF, Briggs WA, Lynne-Davies P, Mcdonald FD. Hypoxemia during hemodialysis using acetate versus bicarbonate dialysate. Am. J. Nephrol. 1984; 4: 248-253.

8. Jacob AI, Gavellas G, Zarco R, Perez G, Bourgiognie JJ. Leukopenia, hypoxia and compliment function with different hemodialysis membranes. Kidney International 1980; 18: 505-509.

9. Francos GC, Besarab A, Burke J, Peters J, Tahamont MV, Gee MH, Flynn JT, Gzesh D. Dialysis-Induced Hypoxemia: Membrane Dependent and Membrane Independent Causes. American Journal of Kidney Diseases, 1985 March; V(3): 191-198.

10. De Backer W, Verpooten GA, Borgonjon Dj, Vermeire PA, Lins RR, De Broe ME. Hypoxemia during hemodialysis: Effects of different membranes and dialysate compositions. Kidney International 1983; 23: 738-743.

11. Igarashi H, Kioi S, Gejyo F, Arakawa M. Physiologic Approach to Dialysis-Induced Hypoxemia: Effects of Dialyzer Material and Dialysate Composition. Nephron. 1985; 41: 62-69. 
12. Dumler F, Levin NW. Leukopenia and Hypoxemia. Arch Intern Med 1979 Oct; 139: 1103-1106.

13. Aurigemma NM, Feldman NT, Gottlieb M, Ingram RH JR, Lazarus JM, Lowrie EG. Arterial Oxygenation During Hemodialysis. N Engl J MED. 1977; 297: 871-973.

14. Craddock PR, Fehr J, Dalmasso AP, Brigham KL, Jacob HS. Hemodialysis Leukopenia: Pulmonary Vascular Leukostasis Resulting from Complement Activation by Dialyzer Cellophane Membranes. The Journal of Clinical Investigation 1977 May; 59: 879-888.

15. Bischel MD, Scoles BG, Mohler JG. Evidence for Pulmonary Microembolization during Hemodialysis. Chest 1975; 67: 335-337.

16. Global Initiative for Chronic Obstructive Lung Disease. A collaborative project of the National Heart Lung and Blood Institute, NIH (USA) and the World Health Organization. Global Initiative for Chronic Obstructive Lung Disease, 2006. http://www.goldcopd.com (last accessed Aug. 9, 2011).

17. Serrano RP, Vega FF, Grande JA. Hypoxemia during hemodialysis in patients with impairment in pulmonary function. Nephron 1986; 42: 14-18.

18. Pitcher WD, Diamond SM, Henrich WL. Pulmonary Gas exchange during dialysis in patients with obstructive lung disease. Chest 1989; 96: 1136-1141.

19. TR Davis, BA Young, MS Eisenberg, TD Rea, MK Copass, LA Cobb. Outcome of cardiac arrests attended by emergency medical services staff at community outpatient dialysis centers. Kidney International 2008; 73: 933-939. 